\title{
Polyimide-Coated Fiber Bragg Grating for Relative Humidity Sensing
}

\author{
Yao LIN, Yuan GONG ${ }^{*}$, Yu WU, and Huijuan WU \\ Key Laboratory of Optical Fiber Sensing and Communications (Ministry of Education of China), University of \\ Electronic Science and Technology of China, Chengdu, 611731, China \\ *Corresponding author: Yuan GONGＥ-mail: ygong@uestc.edu.cn
}

\begin{abstract}
A fiber-optic humidity sensor has been fabricated by coating a moisture sensitive polymer film to the fiber Bragg grating (FBG). The Bragg wavelength of the polyimide-coated FBG changes while it is exposed to different humidity conditions due to the volume expansion of the polyimide coating. The characteristics of sensors, including sensitivity, temporal response, and hysteresis, were improved by controlling the coating thickness and the degree of imidization during the thermal curing process of the polyimide. In the relative humidity $(\mathrm{RH})$ condition ranging from $11.3 \% \mathrm{RH}$ to $97.3 \% \mathrm{RH}$, the sensitivity of the sensor was about $13.5 \mathrm{pm} / \% \mathrm{RH}$ with measurement uncertainty of $\pm 1.5 \%$ RH.
\end{abstract}

Keywords: Fiber Bragg grating, humidity sensing, moisture sensitive

Citation: Yao LIN, Yuan GONG, Yu WU, and Huijuan WU, "Polyimide-Coated Fiber Bragg Grating for Relative Humidity Sensing," Photonic Sensors, 2015, 5(1): 60-66.

\section{Introduction}

With the development of industry and agriculture, the requirements for humidity control and sensing in the areas of aerospace, defense, and power industries are increasingly urgent, especially when the key performance such as the environmental adaptability, measurement range, long-term stability, and measurement accuracy are required to be higher. The fiber Bragg grating (FBG) has been extensively investigated and very widely applied in many fields [1-7]. Compared to conventional capacitive and resistive humidity sensors, the FBG coated with polyimide (PI) used for humidity sensing has unique advantages in terms of high temperature resistance, corrosion resistance, high measurement accuracy, miniaturization, etc. [1, 2]. Thus, FBG humidity sensors provide a feasible method to solve problems in the humidity measurement for the flammable defense and petro chemical research, as well as that in the harsh environment with explosive and strong electromagnetic interference.

The fiber Bragg gratings coated with various sensitive materials have been applied to measure different kinds of samples such as hydrogen, and also for ultrasonic $[4,6]$ and humidity sensing $[8,9]$. Some experiments have verified the excellent linear relationship between the relative humidity and Bragg wavelength shift of the FBG coated with the moisture sensitive material, e.g., PI [10]. The temperature and humidity responses of polyimide-coated FBG humidity sensors have been studied by Yeo and other researchers [10-13].

In this work, the coating process for the FBG

Received: 5 August 2014 / Revised version: 9 September 2014

(C) The Author(s) 2014. This article is published with open access at Springerlink.com

DOI: $10.1007 / \mathrm{s} 13320-014-0218-8$

Article type: Regular 
humidity sensor was modified, and the fabrication parameters such as the coating thickness and heating temperature were optimized. The sensing performance of the polyimide-coated FBG humidity sensor was also evaluated in detail.

\section{Sensing structure and setup}

\subsection{Sensing principle}

The structure of the polyimide-coated FBG humidity sensor is given in Fig. 1. According to the coupled theory [14], the Bragg wavelength shift is given by

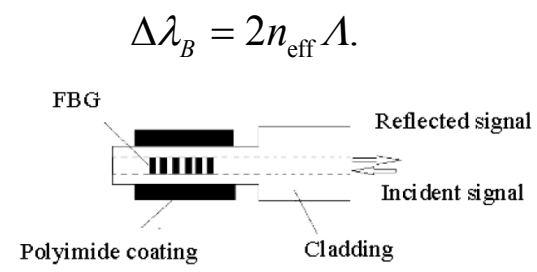

Fig. 1 Structure of the polyimide-coated FBG.

Here, $n_{\text {eff }}$ is the effective refractive index, and $\Lambda$ is the grating pitch. As a function of the change in the strain or thermal effect, the shift in the Bragg wavelength can be expressed as

$$
\frac{\Delta \lambda}{\lambda}=\left(1-P_{e}\right) \varepsilon+\left[\left(1-P_{e}\right) \alpha+\xi\right] \Delta T
$$

where $P_{e}$ represents the photo-elastic constant of the fiber, $\varepsilon$ is the longitudinal strain, and $\zeta$ is the fiber thermo-optical coefficient. The two terms of (2) represent the longitudinal strain effect on the FBG and the thermal effect due to the change in temperature, respectively. In the fixed relative humidity environment, the value of the Bragg wavelength changes due to the thermal effect caused by the change in temperature. As a function of the relative humidity and temperature, the shift in the Bragg wavelength is given by

$$
\frac{\Delta \lambda_{B}}{\lambda_{B}}=C_{1}\left(1-P_{e}\right) \beta \Delta H+\left[C_{1}\left(\alpha_{H}-\alpha_{F}\right)+\xi\right] \Delta T
$$

with

$$
C_{1}=\frac{E_{H}\left(r_{H}^{2}-r_{F}^{2}\right)\left(1-2 \mu_{F}\right)}{\left(1-2 \mu_{H}\right) r_{F}^{2} E_{F}+\left(1-2 \mu_{F}\right)\left(r_{H}^{2}-r_{F}^{2}\right) E_{H}}
$$

where $\alpha_{H}$ is the coefficient of thermal moisture expansion, $\alpha_{F}$ is the thermo-optical coefficient of the single mode fiber, $\beta$ is the coefficient of moisture expansion, $E_{H}$ is Young's modulus, $\Delta T$ and $\Delta H$ are the changes in temperature and relative humidity, $r_{H}$ is the thickness of the polyimide coating, and $r_{F}$ is the radius of the fiber. Equation (3) can be further simplified to be

$$
\frac{\Delta \lambda_{B}}{\lambda_{B}}=K_{T} \Delta T+K_{H} \Delta H
$$

where $K_{T}$ and $K_{H}$ are the temperature and moisture sensitivities of the polyimide-coated FBG. For the FBG without the moisture sensitive material, (4) is simplified to

$$
\frac{\Delta \lambda_{B}}{\lambda_{B}}=K_{T} \Delta T .
$$

By connecting the humidity-sensitive FBG with the FBG temperature sensor in parallel, the temperature effect can be compensated [14]. According to (3), the moisture sensitivity of the polyimide-coated FBG is mainly influenced by two parameters, i.e., the thickness of the polyimide coating and the radius of the fiber.

\subsection{Moisture-sensitive material}

The moisture-sensitive material used in the polymer humidity sensor needs both weak polar groups and a certain amount of hydrophobic groups to balance the requirements for the sensitivity, response speed, wet stagnation, and long-term stability. Thanks to the linear expansion of the PI when exposed to the humidity condition, the PI has been used in different kinds of humidity sensors [ 10 , 12]. The element in the PI adsorbing water is the carboxyl $(-\mathrm{COOH})$ remaining in the imidization process of polyamic acid. The polarity of the residual carboxyl groups left over in the imidization process is weak, which ensures the occurrence of adsorption in the polyimide is reversible [15]. The content of carboxyl in the polyimide is inversely proportional to the humidity sensitivity but proportional to the hysteresis. By studying the 
curing temperature of the PI, we tried to find a reasonable curing process to ensure both the excellent moisture absorption characteristics and moisture desorption characteristics of the polyimide coating.

\subsection{Sensor fabrication}

The Bragg gratings were firstly written into single mode fiber using a phase mask with radiation at the wavelength of $248 \mathrm{~nm}$.

To fabricate the humidity sensors, a thermostable glass mold with a notch in the middle was designed to coat the FBG with a polyimide. The FBG was fixed in the middle of the notch filled with the polyimide acid solution and then cured.

During the curing process of the polyimide solution, the mold was heated, and its loss modulus changed according to the differential scanning calorimetry (DSC) curve in Fig. 2. The polyamic acid started imidization and generated polyimide with the volume reduced down to $15 \%$ of its original volume. Thus, the desired thickness of the PI coating could be precisely controlled by controlling the depth of the notch in the mold. The heat absorption was caused by the evaporation of the water and solvent in the polyamic acid before $250{ }^{\circ} \mathrm{C}$ and by the imidization after $250{ }^{\circ} \mathrm{C}$. After $280{ }^{\circ} \mathrm{C}$, the heat absorption was greatly reduced, which indicated the degree of imidization was highest with few carboxyl groups remained. In our experiment, two different maximum temperatures ( $\left.T_{\text {peak }}\right)$ were applied in the thermal curing process to

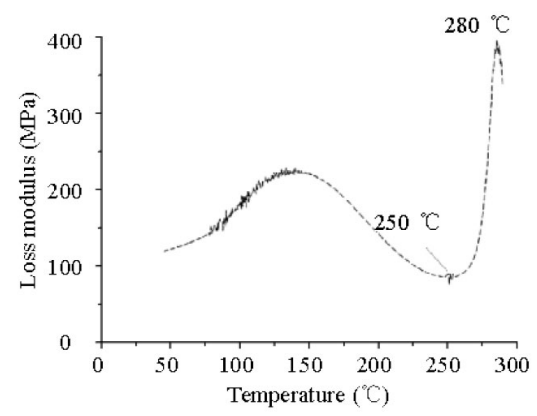

Fig. 2 DSC curve used to control the curing process of the polyamicacid. control the degree of imidization, which helped us explore for the relationship between the curing process and characteristics of polyimide-coated FBG humidity sensor.

\subsection{Experimental setup}

Figure 3 shows the experimental setup for investigating the humidity response with the temperature compensation. The two FBGs were inserted into an airtight glass bottle placed in a thermostatic water tank which could maintain the temperature precisely. To provide a test system of relative humidity, the bottles containing the saturated chemical salt solution were adopted to produce different humidity conditions [15]. A broadband source and an optical spectrum analyzer were connected to two $2 \times 1$ fiber couplers connected in series, as shown in Fig. 3. The optical spectrum analyzer read the wavelength shift of the polyimide-coated FBG while another FBG provided the reference wavelength for the temperature compensation.

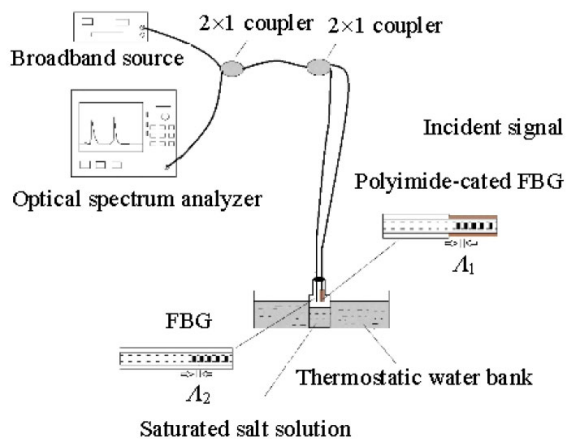

Fig. 3 Experimental setup for the investigation into sensor' humidity response.

\subsection{Calibration}

As a widely applicable method in humidity calibration, the chemical saturated salt solution provides an effective method in the preliminary investigation.

Nine different kinds of chemical saturated salt solutions were adopted to generate the relative humidity conditions at $11.3 \% \mathrm{RH}$ (relative humidity), $22.5 \% \mathrm{RH}, 32.8 \% \mathrm{RH}, 43.2 \% \mathrm{RH}, 54.38 \% \mathrm{RH}$, 
$75.3 \% \mathrm{RH}, 84.2 \% \mathrm{RH}$, and $97.3 \% \mathrm{RH}$, which are listed in Table 1. Despite the lack of controlling humidity continuously, the nine humidity data points listed in Table 1 were enough for the calibration.

Table 1 Relative humidity of the saturated salt solution at $25{ }^{\circ} \mathrm{C}$.

\begin{tabular}{cc}
\hline Chemical symbol & $\mathrm{RH}$ \\
\hline $\mathrm{LiCl}$ & $11.3 \%$ \\
$\mathrm{CH}_{3} \mathrm{COOK}$ & $22.5 \%$ \\
$\mathrm{MgCl}_{2}$ & $32.8 \%$ \\
$\mathrm{~K}_{2} \mathrm{CO}_{3}$ & $43.2 \%$ \\
$\mathrm{Mg}\left(\mathrm{NO}_{3}\right)_{2}$ & $54.38 \%$ \\
$\mathrm{NaCl}$ & $75.3 \%$ \\
$\mathrm{KCl}$ & $84.2 \%$ \\
$\mathrm{~K}_{2} \mathrm{SO}_{4}$ & $97.3 \%$ \\
\hline
\end{tabular}

\section{Results and discuss}

\subsection{Time response}

In all experiments in this work, the sensors were given enough time to equilibrate at $97.3 \% \mathrm{RH}$ in the glass bottle containing the saturated $\mathrm{K}_{2} \mathrm{SO}_{4}$ solution, and then the humidity was decreased step by step to $11.3 \% \mathrm{RH}$ of the saturated $\mathrm{LiCl}$ solution. When exposed to a higher $\mathrm{RH}$ condition, the polyimide adsorbed water and expanded. Thus, the central wavelength of the FBG increased rapidly with time. Adsorbing water molecules continuously, the polyimide became weak in the adsorption capacity of water molecules. As a result, the increasing rate of the wavelength slowed down and eventually reached to equilibrium. The opposite trend curve is showed in Fig. 4(b) when the relative humidity changed from lower $(11.3 \% \mathrm{RH})$ value to higher $(97.3 \% \mathrm{RH})$ value. The temporal response of sensors with a total RH change of $86 \% \mathrm{RH}$ is shown in Table 2.

Table 2 Temporal response data of sensors.

\begin{tabular}{ccc}
\hline $\begin{array}{c}\text { Sensor } \\
\text { number }\end{array}$ & $\begin{array}{c}11.3 \% \mathrm{RH}-97.3 \% \mathrm{RH} \\
(\mathrm{min})\end{array}$ & $\begin{array}{c}97.3 \% \mathrm{RH}-11.3 \% \mathrm{RH} \\
(\mathrm{min})\end{array}$ \\
\hline 1 & 29 & 39 \\
2 & 45 & 58 \\
3 & 120 & 150 \\
4 & 22 & 29 \\
\hline
\end{tabular}

The temporal response of the humidity sensor relates to several factors, such as the thickness of the polyimide coating and the diffusion rate of water in the polymer. In Fig. 4(b), Sensors 1 and 3 have coatings with the same thickness of $15 \mu \mathrm{m}$ and during the thermal curing process exposed under different highest temperatures. According to the DSC curve, Sensor 1 cured at a higher temperature of $290{ }^{\circ} \mathrm{C}$ contained less remaining carboxyl groups than Sensor 3 cured at $260{ }^{\circ} \mathrm{C}$. With the humidity changing from $11.3 \% \mathrm{RH}$ into $97.8 \% \mathrm{RH}$, the response time of Sensor 1 was much less than that of Sensor 3. When the humidity change was reversed, the recovery time of Sensor 1 was also less than that of Sensor 3. Similar results were observed for Sensor 4 with Sensor 2. This results indicated that polyimide containing less carboxyl groups was faster in both moisture desorption and moisture absorption. Therefore, setting a higher curing temperature could improve the temporal response of the humidity sensor.

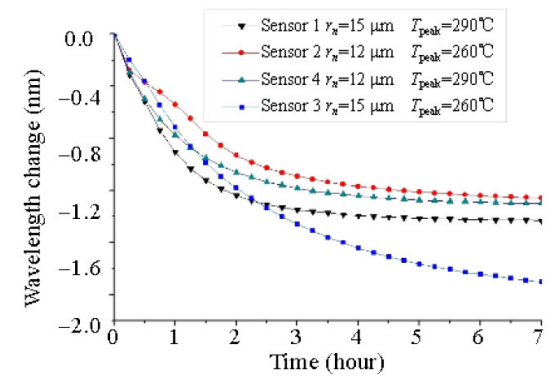

(a)

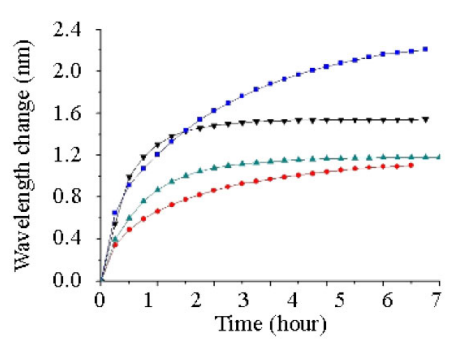

(b)

Fig. 4 Temporal performance of the FBG humidity sensors: (a) temporal responses of sensors with different coating thicknesses and thermal imidization temperatures $\left(T_{\text {peak }}\right)$ when the relative humidity changes from $97.3 \% \mathrm{RH}$ to $11.3 \% \mathrm{RH}$ and (b) temporal responses in the opposite trend.

Despite their identical curing temperature $\left(T_{\text {peak }}=\right.$ 
$260{ }^{\circ} \mathrm{C}$ ), the response time of Sensor 3 with the $15-\mu \mathrm{m}$ thickness coating was longer than that of Sensor 2 with the $12-\mu \mathrm{m}$ thickness coating, as well as Sensor 4 and Sensor 1. Through the above observation, a conclusion can be drawn that the temporal response of the PI coating with the same degree of imidization depends on the thickness of the PI.

\subsection{Hysteresis}

The hysteresis curves of the sensors with different thickness coatings and $T_{\text {peak }}$ are shown in Fig. 5.

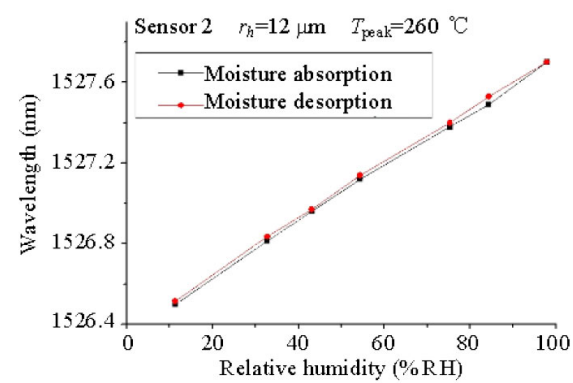

(a)

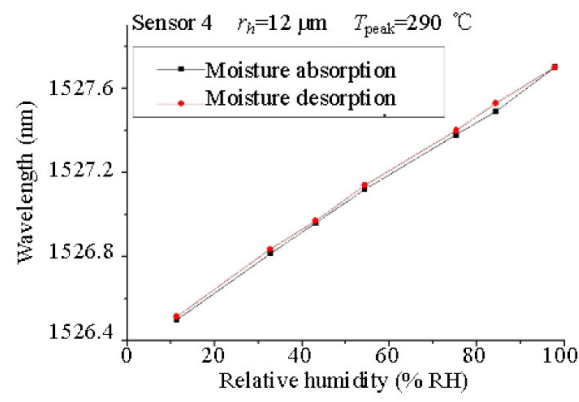

(b)

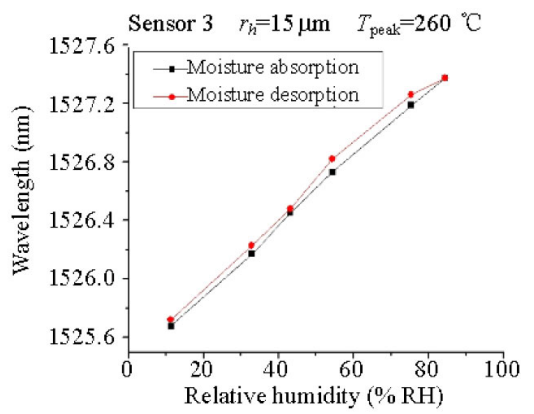

(c)

Fig. 5 Hysteresis curves of the sensors.

An operable method to investigate the hysteresis has been proposed by Yeo et al. [11]. In the experiment, sensors were fixed in the stable humidity environment created by the saturated salt solution at the temperature of $25^{\circ} \mathrm{C}$. The difference between the two curves of Sensor 4 and Sensor 2 with the coating of the same thickness of $12 \mu \mathrm{m}$ was relatively small as shown in Figs. 5(a) and 5(b). The maximum difference $(\sim 1.5 \% \mathrm{RH})$ in Sensor 2 at $54 \% \mathrm{RH}$ was acceptable as well as that in Sensor 4 $(\sim 0.9 \% \mathrm{RH})$ at $44 \% \mathrm{RH}$, which indicated the degree of imidization affected the hysteresis slightly. In Sensor 3 with a thicker coating, the large difference $(\sim 3.5 \% \mathrm{RH})$ revealed that the degree of hysteresis increased due to the thicker coating. Thus, the reliability of sensors which were developed by us depended on the coating thickness largely but on the imidization temperature slightly.

\subsection{Humidity sensitivity}

Thanks to the good linear swollen property of the polyimide, the Bragg wavelength shift is proportional to the change in relative humidity under the constant given temperature condition. The relative humidity sensitivities presented by the slopes of the fitted plots in Fig. 6(a) were $K_{\mathrm{H} 1}=$ $17.8 \mathrm{pm} / \% \mathrm{RH}, \quad K_{\mathrm{H} 2}=12.7 \mathrm{pm} / \% \mathrm{RH}, \quad K_{\mathrm{H} 3}=$ $22.5 \mathrm{pm} / \% \mathrm{RH}$, and $K_{\mathrm{H} 4}=13.5 \mathrm{pm} / \% \mathrm{RH}$, respectively, which indicated the relative humidity sensitivity $K_{\mathrm{H}}$ was affected by both the imidization temperature and thickness of the coating. Obviously with a greater thickness of the coating at a higher imidization temperature, the humidity sensitivity was greater. To investigate the effect of the coating thickness on relative humidity sensitivity, several sensors produced with different thickness coatings but high degree of imidization were measured at the fixed temperature of $25^{\circ} \mathrm{C}$. Figure $6(\mathrm{~b})$ shows the RH sensitivity as a function of the coating thickness. The deviation from the linear relationship might be the result of the unevenness of the contact between the coating and the fiber. The wavelength resolution of the FBG interrogator used in all experiments was $0.001 \mathrm{~nm}$. Thus, the calculated detection limits of Sensor 1 and Sensor 3 were $0.056 \% \mathrm{RH}$ and 
$0.044 \% \mathrm{RH}$, respectively.

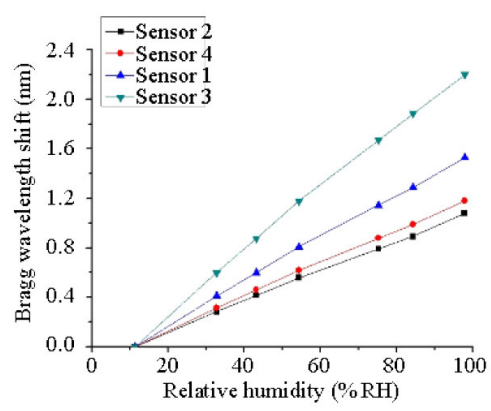

(a)

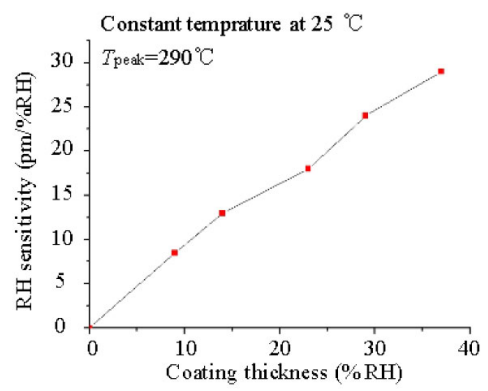

(b)

Fig. 6 Sensitivity of the FBG humidity sensor: (a) Bragg wavelength shift of the sensors with different imidization temperatures and with coating of different thicknesses versus $\mathrm{RH}$ at $25^{\circ} \mathrm{C}$ and (b) $\mathrm{RH}$ sensitivity of sensors with various coating thicknesses.

With a constant $\mathrm{RH}$, the Bragg wavelength changes with temperature with a good linearity, as shown in Fig. 7. The temperature sensitivity kept stable at $24.5 \mathrm{pm} /{ }^{\circ} \mathrm{C}$ in humidity conditions ranging from $11.3 \% \mathrm{RH}$ to $84.4 \% \mathrm{RH}$, but increased to $27.5 \mathrm{pm} /{ }^{\circ} \mathrm{C}$ in the humidity condition above $84.4 \% \mathrm{RH}$, which resulted in an relatively larger error of $6.5 \% \mathrm{RH}$ without the introduction of temperature compensation.

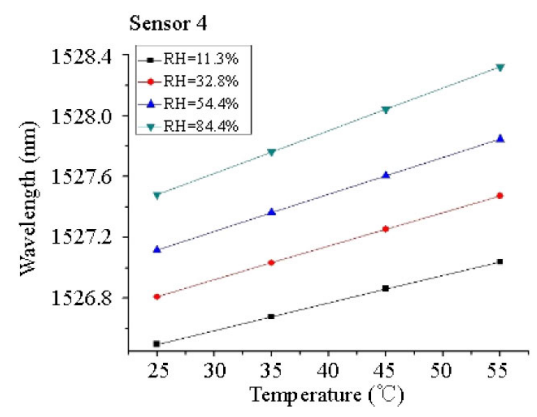

Fig. 7 Bragg wavelength of Sensor 4 against the condition temperature in different $\mathrm{RH}$ conditions.

\subsection{Comprehensive performance}

The response of Sensor 4 against $\mathrm{RH}$ at $25{ }^{\circ} \mathrm{C}$ is given in Fig. 8. The linearity and humidity sensitivity were $0.998 \mathrm{pm} / \% \mathrm{RH}$ and $13.6 \mathrm{pm} / \% \mathrm{RH}$, respectively. Taking both the hysteresis and fitting error into consideration, the maximum error of the Sensors 4 was $1.5 \% \mathrm{RH}$ in the humidity conditions ranging from $11.3 \% \mathrm{RH}$ to $97.3 \% \mathrm{RH}$.

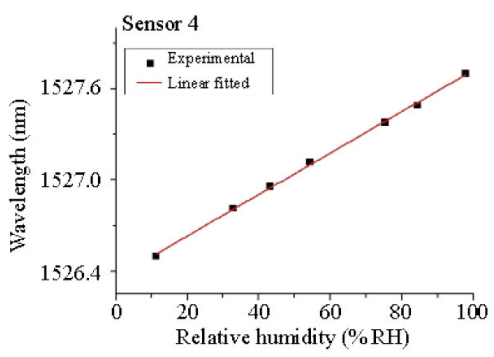

Fig. 8 RH response of Sensor 4 at $25{ }^{\circ} \mathrm{C}$.

\section{Conclusions}

The sensing characteristics of polyimide-coated FBG humidity sensors were studied. In a fixed temperature condition, the wavelength shift of the polyimide-coated FBG humidity sensor kept good linear relationship with the amount of change in the relative humidity. A higher temperature for polyimide acid curing reduced both the response time of sensors and the error produced by the hysteresis. The thicker the polyimide coating was, the higher the humidity sensitivity and reading accuracy were, however, with worse hysteresis and longer response time. The temperature sensitivity of polyimide-coated FBG kept stable under $80 \% \mathrm{RH}$, but increased above $80 \% \mathrm{RH}$, which revealed the necessity of the introduction of temperature compensation to reduce the error.

The polyimide-coated FBG humidity sensor developed in this work could measure the $\mathrm{RH}$ ranging from $11.3 \% \mathrm{RH}$ to $97.3 \% \mathrm{RH}$, with the maximum measuring error of about $1.5 \% \mathrm{RH}$, reading resolution of $0.07 \mathrm{pm} / \% \mathrm{RH}$, and response time of 22 minutes. 


\section{Acknowledgment}

This work is supported by National Natural Science Foundation of China (61107073, 61107072 and 61290312), Fundamental Research Funds for the Central Universities (ZYGX2011J002), Research Fund for the Doctoral Program of Higher Education of China (20110185120020), Program for Changjiang Scholars and Innovative Research Team in University (PCSIRT, IRT1218), and the 111 Project (B14039).

Open Access This article is distributed under the terms of the Creative Commons Attribution License which permits any use, distribution, and reproduction in any medium, provided the original author(s) and source are credited.

\section{References}

[1] Y. J. Rao, "Recent progress in applications of in-fibre Bragg grating sensors," Optics and Lasers in Engineering, 1999, 31(4): 297-324.

[2] Y. J. Rao, "In-fiber Bragg grating sensors," Measurement Science and Technology, 1997, 8(4): 355-375.

[3] B. Sutapun, M. Tabibazar, and A. Kazemi, "Pd-coated elastooptic fiber optic Bragg grating sensors for multiplexed hydrogen sensing," Sensors and Actuators B: Chemical, 1999, 60(1): 27-34.

[4] W. Li, J Pang, X. Lu, and J. Liu, "Rail expansion devices monitored by FBG sensors on an urban railway viaduct," Photonic Sensors, 2014, 4(2): 173-179.

[5] D. Pang and Q. Sui, "Response analysis of ultrasonic sensing system based on fiber Bragg gratings of different lengths," Photonic Sensors, 2014, 4(3): 281-288.

[6] S. Saad, L. Hassine, and W. Elfahem, "Hydrogen FBG sensor using $\mathrm{Pd} / \mathrm{Ag}$ film with application in propulsion system fuel tank model of aerospacevehicle," Photonic Sensors, 2014, 4(3): 254-264.

[7] J. G. V. Teixeira, I. T. Leite, S. Silva, and O. Frazão, "Advanced fiber-optic acoustic sensors," Photonic Sensors, 2014, 4(3): 198-208.

[8] K. M. Tan, C. M. Tay, S. C. Tjin, C. C. Chan, and H. Rahardjo, "High relative humidity measurements using gelatin coated long-period grating sensors," Sensors and Actuators B: Chemical, 2005, 110(2): 335-341.

[9] X. F. Huang, D. R. Sheng, K. F. Cen, and H. Zhou, "Low-cost relative humidity sensor based on thermoplastic polyimide-coated fiber Bragg grating," Sensors and Actuators B: Chemical, 2008, 127(2): 518-524.

[10] J. Y. Jun, L. H. Bao, and H. Liu, "Sketch of moisture sensitive polymer use with fiber Bragg grating based humidity sensor," College Physics, 2009, 28(7): $39-42$.

[11] T. L. Yeo, T. Sun, K. T. V. Grattan, D. Parry, R. Lade, and B. D. Powell, "Polymer coated fiber Bragg grating for relative humidity sensing," IEEE Sensors Journal, 2005, 5(5): 1082-1089.

[12] B. Adhikari and S. Majumdar, "Polymers in sensor applications," Progress in Polymer Science, 2004, 29(7): 699-766.

[13] A. P. Russell and K. S. Fletcher, "Optical sensor for the determination of moisture," Analytica Chimica Acta, 1985, 170: 209-216.

[14] K. O. Hill and G. Meltz, "Fiber Bragg grating technology fundamentals and overview," Journal of Lightwave Technology, 1997, 15(8): 1263-1276.

[15] US Standard, ASTME104-02, Standard Practice for Maintaining Constant Relative Humidity by Means of Aqueous Solutions. 